ISSN 0280-5316 ISRN LUTFD2/TFRT--5720--SE

\title{
Analysis of Interconnected Systems
}

Ather Gattami

Department of Automatic Control Lund Institute of Technology August 2003 



\begin{tabular}{|c|c|c|c|}
\hline \multirow{3}{*}{\multicolumn{2}{|c|}{$\begin{array}{l}\text { Department of Automatic Control } \\
\text { Lund Institute of Technology } \\
\text { Box } 118 \\
\text { SE-221 } 00 \text { Lund Sweden }\end{array}$}} & \multicolumn{2}{|c|}{$\begin{array}{l}\text { Document name } \\
\text { MASTER THESIS }\end{array}$} \\
\hline & & \multicolumn{2}{|l|}{$\begin{array}{l}\text { Date of issue } \\
\text { August } 2003\end{array}$} \\
\hline & & \multicolumn{2}{|c|}{$\begin{array}{l}\text { Document Number } \\
\text { ISRN LUTFD2/TFRT--5720--SE }\end{array}$} \\
\hline \multirow{2}{*}{\multicolumn{2}{|c|}{$\begin{array}{l}\text { Author }(s) \\
\text { Ather Gattami }\end{array}$}} & \multicolumn{2}{|c|}{$\begin{array}{l}\text { Supervisor } \\
\text { Anders Rantzer Reglerteknik, LTH } \\
\text { Richard Murray Caltech, USA }\end{array}$} \\
\hline & & \multicolumn{2}{|c|}{ Sponsoring organization } \\
\hline \multicolumn{4}{|c|}{$\begin{array}{l}\text { Title and subtitle } \\
\text { Analysis of Interconnected Systems. (Analys av kopplade system) }\end{array}$} \\
\hline \multirow{2}{*}{\multicolumn{4}{|c|}{$\begin{array}{l}\text { Abstract } \\
\text { In this paper analysis of interconnected dynamical systems are considered. A solid framework for the analysis of the } \\
\text { stability of interconnection is given. The results from [1] that studies the SISO-case for a constant interconnection matrix } \\
\text { are generalized to the MIMO-case where arbitrary interconnection is allowed. The analysis show existence of a separation } \\
\text { principle that is very useful in the sense of the simplicity for stability analysis. Stability could be checked graphically } \\
\text { using a Nyquist-like criterion. The problem with time-delays and interconnection variation and robustness appear to be } \\
\text { natural special cases of the general framework. }\end{array}$}} \\
\hline & & & \\
\hline \multicolumn{4}{|c|}{ Classification system and/or index terms (if any) } \\
\hline \multicolumn{4}{|c|}{ Supplementary bibliographical information } \\
\hline $\begin{array}{l}I S S N \text { and } \\
0280-53\end{array}$ & & & $I S B N$ \\
\hline $\begin{array}{l}\text { Language } \\
\text { English }\end{array}$ & $\begin{array}{l}\text { Number of pages } \\
37\end{array}$ & \multirow[t]{2}{*}{ Recipient's notes } & \\
\hline Security $\mathrm{cl}$ & & & \\
\hline
\end{tabular}





\section{Contents}

1 Introduction $\quad 5$

1.1 Motivation .................. 5

1.2 Previous Work .................. 5

1.3 Outline of the paper .............. 6

2 Preliminaries $\quad 7$

2.1 Notation . . . . . . . . . . . . . . . 7

2.2 Matrix Algebra . . . . . . . . . . . . . 7

2.3 Two Results from System Theory . . . . . . . . . . . . . 8

2.4 Algebraic Graph Theory . . . . . . . . . . . . . . . 9

3 Main Results 12

3.1 Stability of Interconnection Represented by Feedback Transfer Matrix .................... 12

3.2 Robustness . . . . . . . . . . . . . . 15

4 Applications $\quad 20$

4.1 The Consensus Problem . . . . . . . . . . . . . 20

4.2 Stable SISO Plants and Interconnection . . . . . . . . . . . 21

4.3 Interconnection with Fixed Time-delays . . . . . . . . . . . 22

4.4 Interconnection with Random Delays . . . . . . . . . . . 22

4.5 Time-varying Interconnection . . . . . . . . . . . . . 24

4.6 A Numerical Example . . . . . . . . . . . . . . . . . . 26

5 Conlclusions and Future Work 30

$\begin{array}{lr}\text { A Matlab-files } & 31\end{array}$

A.1 Simulink Models . . . . . . . . . . . . . . . . . . 31

A.2 Matlab Code . . . . . . . . . . . . . . . . 31 



\section{Acknowledgements}

The road leading to the paper you are reading has been full of both happy and painfull moments.

I was supposed to start my Master's thesis in September 2003 at Caltech, USA. But unfortunately my visa application got delayed three months, so I started at Caltech in late January 2003. During this hard time of uncertainty I got invaluable support from the Department of Automatic Control in Lund. Therefore I would like to thank everyone at the department for their care and kindness. In particular my deepest gratitudes goes to the golden lady Eva Schildt, for spending too much time with my complicated application procedures. Eva, I will never forget your help. I would also like to thank Professor Per Hagander for taking his time reading letters that I wrote to the US embassy in Stockholm.

Many thanks to the nice secretaries Britt-Marie and Agneta for waiting too long for this manuscirpt to be finished!

I am very grateful to Professor Anders Rantzer for being supportive not only during doing my Master's work but also during my undergraduate studies.

I have spent five wonderful months at Caltech thanks to many people. First of all I would like to thank my supervisor Professor Richard M. Murray who has been a great teacher and friend. His kindness is beyond what I could express with words.

I had many interesting discussions with Reza Olfati-Saber and Vijay Gupta. Talking with them expanded my view on many aspects of my Master work. Alex Fax was very kind to send me his Matlab-files to use and modify, and I am grateful to his help.

My great office-mates Melvin Flores and Alfredo Martinez played a big role in my everyday life in Pasadena. Melvin was my faithfull lunch partner and I had a lot of unforgettable moments with him(especially taking the course Geometry of Nonlinear Systems). He had to stand with my complains and did honest efforts to ease my worries. By the way Melvin, are you up for lunch?

Alfredo has been one of my best friends. He was really the one that I could turn to when I needed help. I haven't met so many people with such a noble heart. I would also like thank Andrew, Brad and Kevin for being great friends, and we had a lot of fun together. 
My family has been, and still is, my main source of energy and without them this work wouldn't be possible.

Finally I would like to thank my great friends who sent to me e-mails continuously making me feel their presence. I consider myself lucky to have you around me!

Ather Gattami, January 2004, Sweden. 


\section{Introduction}

\subsection{Motivation}

In recent years there has been a large amount of interest in analysis of interconnected systems and networks, where the relation between the interconnection and stability of the resulting systems are related. In particular, there has been an attempt to focus on distributed systems where the controller is decentralized, i.e each plant of the interconnected system makes a decision based on limited information that might be available to it.

Interconnection can be found in our everyday life. There are many examples of such systems, and here we give only a sample of different problems that have the issue of interconnection in common. The internet is a very large network where stability issues are of great interest. The information flow transported along different links could, e.g., be delayed which makes it hard to stabilize the entire network if the delays are not taken into account.

Economy markets are another example of rather complicated pricing system where we do have a lot of manual control, and in the same time a lot of interconnection between different pricing dynamics.

The power network is probabely one of the most complex networks. We can find stability problems not only when trying to robustly stabilize the

physical power network(which is hard enough), but also stabilize the econimcs marked that is embedded into it.

Consumers, distributers, and power generators try to optimize their profit. Therefore, we have to take into account the economics network that also could be unstable, where the pricing plays a large role. An example is the California power crisis of 2000.

In later years, even stability of vehicle formations has been of great interest, e.g. formation of unmanned air vehicles (UAV), robots, and sattelites are only few examples.

\subsection{Previous Work}

There has been a lot of research on interconnected systems where some focused on particular "real-world" problems and some on trying to find a more general approach to analyze interconnected systems and give a constructive way for designing the decetralized controller.

In Fax and Murray [1] a nyquist-like criterion is derived for stability check under a constant feedback matrix for SISO systems. Also a sufficient condition is given for interconnected MIMO systems. In OlfatiSaber and Murray [2] the average-consensus problem was considered 
for the case of single integrators. Briefly, the average-consensus problem about trying to make a group of plants agree on the average of their states or outputs under some interconnection between different plants. Also, [2] touches the idea of introducing an interconnection matrix that is frequency dependent and examine its eigenvalues to derive stability conditions. The work by V. Gupta et al[3] derives stability conditions for stochastically varying interconnection. In A. Jadababaie et al[4] the problem with switched interconnection is also considered for the case where the switching rule are restricted to certain properties. M. Rotkowitz et al [7] introduces the notion of quadratic invariance and how it could be used in constructing a descentralized control law by minimizing the closed-loop norm of feedback system subject to constraints on the controller structure. G. Vinnicombe [6] considers the effect of time-delays in the stability of end-to-end congestion control for the internet.

Initially, the problem of how time-delays affected stability of vehicle formations was considered, building on the work done in [1]. Trying to find an approach to solve the problem, the framework given in [1] needed to be extended, starting with translating the problem formulation from the time-domain to the frequency domain. The new formulation of the problem was one of the vital parts of this thesis. Interesting properties showed up and proved to be very useful for other kind of problems.

Hence the main goal of the thesis is to introduce a general framework for interconnected systems where we try to include all problems discussed above and state them in a simple and classical form, which hopefully reveals many properties that give us an easy way of stability analysis and system design. Here we try to show how the problem with time-varying connections and delays could be easly modelled using the general framework. Also we show a Nyquist-like criterion inspired by the one in [1], that could ease the analysis of the interconnection.

\subsection{Outline of the paper}

We introduce notation and some mathematical tools in Chapter 2. In Chapter 3 we give the problem setup and the framework used throughout the thesis. We also introduce the main theorems. Chapter 4 show how we could model different problems in way that resembles the general framework. We discuss a numerical problem more deeply to show a possible application of the the main result. We finally discuss the resluts in this paper and suggest future directions related to the thesis in Chapter 5. In the appendix we list the Matlab-files for the simulations and other stablility tools for the Nyquist-like plots. 


\section{Preliminaries}

In this section we introduce some mathematical results that are used throughout the paper.

\section{$2.1 \quad$ Notation}

We denote a set of elements $\left\{a_{1}, a_{2}, \ldots, a_{n}\right\}$ by $\left\{a_{i}\right\}^{n}$ or more briefly $\left\{a_{i}\right\}$ if the number of elements is clear from the context. We define $A^{T}$ and $\bar{A}$ as the transpose and complex conjugate of the matrix $A$ respectively. Also let $A^{*}$ be the Hermitian matrix of $A$, i.g.

$$
A^{*}=\bar{A}^{T} \text {. }
$$

\subsection{Matrix Algebra}

A matrix $U$ is said to be unitary if

$$
U^{*}=\bar{U}^{T}=U^{-1} .
$$

The Kronecker product for two matrices $A$ and $B$ is defined as

$$
A \otimes B=\left(\begin{array}{cccc}
A_{11} B & A_{12} B & \ldots & A_{1 l} B \\
A_{21} B & A_{22} B & \ldots & A_{2 l} B \\
\vdots & \vdots & \ddots & \vdots \\
A_{k 1} B & A_{k 2} B & \ldots & A_{k l} B
\end{array}\right)
$$

For a set of $N$ matrices $\left\{M_{1}, \ldots, M_{N}\right\}$ of size $r \times s$, we define the direct sum as the $N r \times N s$ blockdiagonal matrix $\widehat{M}$ whose $r \times s$ diagonal blocks are the matrices $M_{1}, \ldots, M_{n}$ (in this order), and the other entries are zero, which we write as

$$
\widehat{M}=\oplus \sum_{i=1}^{N} M_{i}
$$

For a given $N \times N$ matrix $Q$, define an $N k \times N k$ matrix $Q_{(k)}$ by the equation

$$
Q_{(k)}=Q \otimes I_{k}
$$

where $I_{k}$ is the $k \times k$ identity matrix. So, we have simply replaced each element of $Q$ by the same element times the $k \times k$ identity matrix. Given an $n \times n$ complex matrix $A$ with eigenvalues $\left\{\lambda_{i}\right\}$ in any prescribed order, there is a unitary matrix $U$ such that $U^{*} A U=T$ where $T$ is upper triangular with its diagonal elements $T_{i i}=\lambda_{i}$. This is called the Schur triangular factorization. The singular values $\left\{\sigma_{i}\right\}$ 
of a matrix $A$ are the positive square roots of the eigenvalues of $A^{*} A$, i.e. $\sigma_{i}=\sqrt{\lambda_{i}^{*} \lambda}$. The $\mathcal{H}_{\infty}$-norm of matrix $A$ is defined as the largest singular value of $A$, i.e

$$
\|A\|_{\infty}=\bar{\sigma}=\max \left\{\sigma_{i}\right\}
$$

Now we state the Geršgorin disc theorem(for a proof consult e.g. [13]):

Proposition 1. Let $A=\left[A_{i j}\right]$ be an $n \times n$ matrix, and let

$$
C_{j}(A)=\sum_{i=1, i \neq j}^{n}\left|A_{i j}\right| .
$$

Then all eigenvalues of $A$ are located in the union of $n$ discs

$$
\bigcup_{j=1}^{n}\left\{z \in \mathbb{C}:\left|z-A_{j j}\right| \leq C_{j}\right\} .
$$

\subsection{Two Results from System Theory}

A MIMO system that is described by a square transfer matrix $G(s)$ is said to be stable if and only if $\operatorname{det}[G(s)]$ has no eigenvalues in the right half of the complex plane (RHP).

\section{The Generalized Nyquist Theorem:}

Let $G(s)$ be a transfer square matrix. Let $p$ be the number of openloop unstable poles in $G(s)$. The closed-loop system with loop transfer matrix $G(s)$ and negative feedback is stable if and only if the Nyquist plot of $\operatorname{det}(I+G(s))$

i) makes $p$ anti-clockwise encirclements of the origin, and

ii) does not pass through the origin.

\section{The Small Gain Theorem:}

Let $G(s)$ be a stable transfer square matrix with

$$
\|G(s)\|_{\infty} \leq \gamma .
$$

The closed-loop system with loop transfer matrix $G(s)$ and negative feedback matrix $\Delta(s)$ is stable if

$$
\|\Delta(s)\|_{\infty}<\frac{1}{\gamma}
$$




\subsection{Algebraic Graph Theory}

Here we introduce some results from algebraic graph theory. Some of the results presented are not used in this paper, but shows many interesting relations that actually have been used recently in the context of networks and stabilization of vehicle formations.

A (simple) graph $\mathcal{G}$ is a mathematical structure that consists of finite set of elements $\mathcal{V}=\left\{v_{1}, v_{2}, \ldots, v_{n}\right\}$ called vertices, or nodes, with a prescribed set $\mathcal{E}$ of unordered pairs of distinct vertices of $\mathcal{V}$. Every element $e \in \mathcal{E}$ can be written as $e=\left(v_{i}, v_{j}\right), v_{i}, v_{j} \in \mathcal{V}$, and $e$ is called an edge, or arc, of the graph $\mathcal{G}$. We call $v_{i}$ and $v_{j}$ the endpoints of $e$. We say that $v_{i}$ and $v_{j}$ are connected if $\left(v_{i}, v_{j}\right) \in \mathcal{E}$. We infer also the notion of a directed edge $e_{i j}=\left(v_{i}, v_{j}\right)$, which could be considered geometrically as an arrow from the node $v_{i}$ to $v_{j}$. A graph with directed edges is called a directed graph. A graph where $e_{i j} \in \mathcal{E} \leftrightarrow e_{j i} \in \mathcal{E}$ is called undirected graph. The number $n$ of elements of the finite set $\mathcal{V}$ is called the order of the graph $\mathcal{G}$. Two vertices on the same edge or two edges with a common vertex are adjacent. A graph $\mathcal{K}$ is called complete if all possible pairs of vertices are connected. If we allow a pair of vertices to form more than one distinct edge, the structure is called a multigraph. If we allow edges of the form $\left(v_{i}, v_{i}\right)$, we get loops, and we have a general graph. If two edges have a common vertex, then they are said to be incident with this vertex. The degree of a vertex, or valency, is the number of incident edges with the vertex. The graph $\mathcal{G}$ is called regular if all vertices have the same degree. If the each vertex has degree $r$ in a graph, then we say that the graph is regular with order $k$. For a general directed graph $\mathcal{G}$ with vertices $\mathcal{V}=\left\{v_{1}, v_{2}, \ldots, v_{n}\right\}$ we define the multiplicity of a pair of vertices $\left(v_{i}, v_{j}\right)$ as $\operatorname{mul}\left(v_{i}, v_{j}\right)$ which is the number of edges from $v_{i}$ to $v_{j}$. For example, $\operatorname{mul}\left(v_{i}, v_{i}\right)$ denotes the number of loops at vertex $v_{i}$, and for a simple graph $\operatorname{mul}\left(v_{i}, v_{j}\right)=1$ for all pair of vertices $\left(v_{i}, v_{j}\right)$. Consider a matrix $A$ such that that the element $a_{i j}$ is equal to the multiplicity of an edge $\left(v_{i}, v_{j}\right)$. Then $A$ is a matrix of order $n$ and is called the adjacency matrix of $\mathcal{G}$. Let $\mathcal{G}$ be a general graph of order $n$ with the set of vertices $\mathcal{V}=v_{1}, v_{2}, \ldots v_{n}$ and set of edges $\mathcal{E}=e_{1}, e_{2}, \ldots e_{m}$. Now we construct the incidence matrix $B$ for the graph $\mathcal{G}$ in the following way: the element $b_{i j}$ in position $(i, j)$ in $B$ is set equal to 1 if vertex $v_{j}$ is on edge $e_{i}$ and 0 otherwise. Each row of $B$ contains at least one entry equal to one and not more than two 1 's as entries. The rows with single 1 in $B$ correspond to the edges that are loops in $\mathcal{G}$. It is also easy to see that identical rows in $B$ correspond to multipule edges in $\mathcal{G}$.

There is a very elegant realtionship between the adjacency and incidence matrix of a graph: 
Propostion 2. Let $\mathcal{G}$ be a multigraph of order $n$. Let $A$ be the adjacency matrix of $\mathcal{G}$ and let $B$ be the incidence matrix of $\mathcal{G}$. Then

$$
B^{T} B=A+D
$$

where $D$ is a diagonal matrix of order $n$ whose diagonal entry $d_{i}$ is the degree of the vertex $v_{i}$ of $\mathcal{G}, i=1,2, \ldots, n$.

Proof. The proof is simple. It is not hard to see that for $i \neq j$, the scalar product of columns $i$ and $j$ of $B$ equals $\operatorname{mul}\left(v_{i}, v_{j}\right)$. The scalar product of column $i$ of $B$ with itself is equal to the degree of the vertex $v_{i}, \mathcal{Q} . \mathcal{E} . \mathcal{D}$.

A matrix $A$ is positive (nonnegative) if each entry in $A$ is positive (nonnegative).

Let $A$ and $B$ be two nonnegative $n \times n$ matrices. We write $A>B$ ( $A \geq B$ ) if $A-B$ is positive (nonnegative).

A graph $\mathcal{G}$ with adjacency matrix $A$ is isomorphic to another graph $\mathcal{G}^{\prime}$ if there is exists a permutation matrix $P$ such that

$$
P A P^{T}=A^{\prime}
$$

The matrix A is said to be reducible if there exists a permutation matrix $P$ such that

$$
P A P^{T}=\left[\begin{array}{cc}
E & F \\
0 & G
\end{array}\right]
$$

where $E$ and $G$ are square matrices. If $A$ is not reducible, then it is said to be irreducible.

Proposition 3. The adjacency matrix $A$ is irreducible iff its corresponding graph $\mathcal{G}$ is strongly connected.

Proof. If $A$ is reducible, then from (2) we see that the vertices can be divided in two subsets; one subset belongs to the rows of $E$ and the other belongs to the rows of $G$. The latter subset is closed, because there is no walk from the second subset to the first one. Hence, the graph is not strongly connected.

Now, suppose that $\mathcal{G}$ is not strongly connected. Then there exsists an isolated subset of vertices. Permute the vertices of $\mathcal{G}$ such that the vertices in the isolated subset comes last in the enumeration of $\mathcal{G}$. Then we see that the same permutation with $A$ gives a block-triangular form as in (2). 
Proposition 4. The adjacency matrix $A$ of order $n$ of the graph $\mathcal{G}$ is irreducible iff

$$
(A+I)^{n-1}>0
$$

Proof. We have by the binomial theorem

$$
(A+I)^{n-1}=\sum_{k=0}^{n-1}\left(\begin{array}{c}
n-1 \\
k
\end{array}\right) A^{k}
$$

The entry in position $(i, j)$ on the LHS of $(3)$ is positive iff the corresponding entry in at least one of the matrices $I, A, \ldots, A^{n-1}$ in the RHS of (3) is postive. But this holds iff there exists a walk of length $\leq n-1$ from $v_{i}$ to $v_{j}$. Because the shortest walk includes each vertex not more than once, then there is a walk of length $<n$. We conclude that the $A$ is irreducible exactly when the matrix on the LHS is positive, $\mathcal{Q} . \mathcal{E} . \mathcal{D}$.

There is a special graph that has been used frequently in connection with modelling of networks. Let $D$ be a diagonal matrix with $D_{i i}$ equal to the out-degree of vertex $i$. The Laplacian of a graph is defined as

$$
L=D-A .
$$

though we will make a slight modification and define the Laplacian as

$$
L_{i j}= \begin{cases}1 & \text { if } i=j \\ -\frac{1}{\left|\mathcal{J}_{i}\right|} & \forall j \in \mathcal{J}_{i} \\ 0 & \text { otherwise }\end{cases}
$$

or more algebraically

$$
L=D^{-1}(D-A)
$$

assuming that $D_{i i} \neq 0$ for all $i$ (note that the first definition does not require the later condition). The question of which definition to be used depends on the application.

The Laplacian is useful since many studies has been focused on its properties, and especially the spectral properties. 


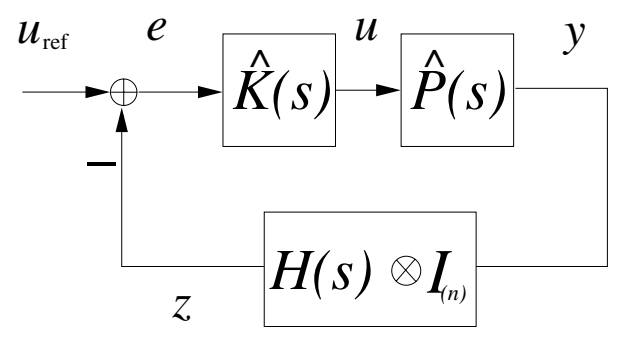

Figure 1: The interconnected system.

\section{$3 \quad$ Main Results}

\subsection{Stability of Interconnection Represented by Feedback Transfer Matrix}

We start by considering a set of $N$ identical plants and its controllers given by the matrix functions $P(s)$ and $K(s)$ of size $n \times m$ and $m \times n$ respectively. Let

$$
\widehat{P}(s)=\oplus \sum_{i=1}^{N} P(s)
$$

and

$$
\widehat{K}(s)=\oplus \sum_{i=1}^{N} K(s)
$$

Now consider the interconnected MIMO system given as in the figure below, where $H(s)$ is the interconnection matrix function with proper

dimensions. We can state a simple stability theorem for the system above, but first we need we need a very useful relation that was first shown in [1]:

Lemma 1. Let $Q$ be a $N \times N$ matrix, $M$ be $r \times s$ matrix with $\widehat{M}$ of size $N r \times N$ s such that $\widehat{M}=I_{N} \otimes M=\operatorname{diag}(M, \ldots, M)$ and let $Q_{(k)}=Q \otimes I_{k}$ where $\otimes$ denotes the Kronecker product, and $I_{k}$ is the $k \times k$ identity matrix. Then

$$
\widehat{M} Q_{(s)}=Q_{(r)} \widehat{M}
$$


Proof. Direct computation gives

$$
\begin{aligned}
\widehat{M} Q_{(s)} & =\left(\begin{array}{cccc}
M & 0 & \ldots & 0 \\
0 & M & \ldots & 0 \\
\vdots & \vdots & \ddots & \vdots \\
0 & 0 & \ldots & M
\end{array}\right)\left(\begin{array}{cccc}
Q_{11} I_{s} & Q_{12} I_{s} & \ldots & Q_{1 N} I_{s} \\
Q_{21} I_{s} & Q_{22} I_{s} & \ldots & Q_{2 N} I_{s} \\
\vdots & \vdots & \ddots & \vdots \\
Q_{N 1} I_{s} & Q_{N 2} I_{s} & \ldots & Q_{N N} I_{s}
\end{array}\right) \\
= & \left(\begin{array}{cccc}
Q_{11} M & Q_{12} M & \ldots & Q_{1 N} M \\
Q_{21} M & Q_{22} M & \ldots & Q_{2 N} M \\
\vdots & \vdots & \ddots & \vdots \\
Q_{N 1} M & Q_{N 2} M & \ldots & Q_{N N} M
\end{array}\right)
\end{aligned}
$$

In the same manner we find that

$$
\begin{aligned}
Q_{(r)} \widehat{M} & =\left(\begin{array}{cccc}
Q_{11} I_{r} & Q_{12} I_{r} & \ldots & Q_{1 N} I_{r} \\
Q_{21} I_{r} & Q_{22} I_{r} & \ldots & Q_{2 N} I_{r} \\
\vdots & \vdots & \ddots & \vdots \\
Q_{N 1} I_{r} & Q_{N 2} I_{r} & \ldots & Q_{N N} I_{r}
\end{array}\right)\left(\begin{array}{cccc}
M & 0 & \ldots & 0 \\
0 & M & \ldots & 0 \\
\vdots & \vdots & \ddots & \vdots \\
0 & 0 & \ldots & M
\end{array}\right) \\
& =\left(\begin{array}{cccc}
Q_{11} M & Q_{12} M & \ldots & Q_{1 N} M \\
Q_{21} M & Q_{22} M & \ldots & Q_{2 N} M \\
\vdots & \vdots & \ddots & \vdots \\
Q_{N 1} M & Q_{N 2} M & \ldots & Q_{N N} M
\end{array}\right)
\end{aligned}
$$

and the proof is complete.

Theorem 2. Let $U(s)$ be a vector of size $m N, Y(s)$ and $Z(s)$ be vectors of size $n N, H(s)$ a matrix of size $N \times N$. Also set

$$
\widehat{P}(s)=\oplus \sum_{i=1}^{N} P(s)
$$

and

$$
\widehat{K}(s)=\oplus \sum_{i=1}^{N} K(s),
$$

where $P(s)$ and $K(s)$ are matrices of size $n \times m$ and $m \times n$ respectively. Let $T(s)=S(s)^{*} H(s) S(s)$ where $S(s)$ is the unitary Schurtransformation matrix such that $T(s)$ is upper triangular with the eigenvalues of $H(s)$ on its diagonal. Let $p$ be the number of unstable poles for $\operatorname{det}\left[T_{(n)}(s) \widehat{P}(s) \widehat{K}(s)\right]$. Then the control law $U(s)=\widehat{K}(s)\left(U_{\text {ref }}-Z(s)\right)$ stabilizes the system

$$
\left\{\begin{array}{l}
Y(s)=\widehat{P}(s) U(s) \\
Z(s)=H_{(n)}(s) Y(s)
\end{array}\right.
$$


iff the Nyquist plot of

$$
\operatorname{det}\left[I_{n N}+T_{(n)}(s) \widehat{P}(s) \widehat{K}(s)\right]
$$

makes $p$ anti-clockwise encirclements of the origin.

Proof. First we notice that

$$
\begin{aligned}
\operatorname{det}\left[H_{(n)}(s) \widehat{P}(s) \widehat{K}(s)\right] & =\operatorname{det}\left[S_{(n)}(s) T_{(n)} S_{(n)}^{*}(s) \widehat{P}(s) \widehat{K}(s)\right] \\
& =\operatorname{det}\left[T_{(n)}(s) \widehat{P}(s) \widehat{K}(s)\right]
\end{aligned}
$$

by applying Lemma 1 and the fact that $\operatorname{det}\left(S_{(n)}\right)=\operatorname{det}\left(S_{(n)}^{*}\right)=1$.

The closed-loop dynamics are given by

$$
\begin{aligned}
Z(s) & =H_{(n)}(s) Y(s) \\
& =H_{(n)}(s) \widehat{P}(s) U(s) \\
& =H_{(n)}(s) \widehat{P}(s) \widehat{K}(s)\left(U_{\mathrm{ref}}-Z(s)\right),
\end{aligned}
$$

which is equivalent to

$$
Z(s)=\left(I_{n N}+H_{(n)}(s) \widehat{P}(s) \widehat{K}(s)\right)^{-1} H_{(n)}(s) \widehat{P}(s) \widehat{K}(s) U_{\text {ref }}(s)
$$

Using the generalized Nyquist Theorem, we see that the closed-loop system is stable iff the Nyquist plot of

$$
\operatorname{det}\left[I_{n N}+H_{(n)}(s) \widehat{P}(s) \widehat{K}(s)\right]
$$

makes $p$ anti-clockwise encirclements of the origin. But using Lemma 1 we get

$$
\begin{aligned}
\operatorname{det}\left[I_{n N}+H_{(n)}(s) \widehat{P}(s) \widehat{K}(s)\right] & =\operatorname{det}\left[I_{n N}+S_{(n)}(s) T_{(n)} S_{(n)}^{*}(s) \widehat{P}(s) \widehat{K}(s)\right] \\
& =\operatorname{det}\left[S_{(n)}\left(I_{n N}+T_{(n)}(s) \widehat{P}(s) \widehat{K}(s)\right) S_{(n)}^{*}\right] \\
& =\operatorname{det}\left[I_{n N}+T_{(n)}(s) \widehat{P}(s) \widehat{K}(s)\right]
\end{aligned}
$$

What is interesting about Theorem 2 is the fact that the analysis for the interconncted system gets very easy, because we get some kind of a separation between the systems. Since $T_{(n)}(s)$ is block upper triangular and both $\widehat{P}(s)$ and $\widehat{K}(s)$ are block diagonal, we get

$$
\operatorname{det}\left[T_{(n)}(s) \widehat{P}(s) \widehat{K}(s)\right]=\prod_{i=1}^{n} \lambda_{i}(s) \operatorname{det}[P(s) K(s)]
$$

where $\lambda_{i}(s)$ denotes the $i$ th diagonal element of $T(s)$, or simply the $i$ th eigenvalue of $H(s)$. Hence, to check the number of unstable poles for $\operatorname{det}\left[T_{(n)}(s) \widehat{P}(s) \widehat{K}(s)\right]$ we simply need to check the unstable poles 
of $P(s) K(s)$ and $\lambda_{i}(s)$ for $i=1, \ldots, n$.

In addition

$$
\operatorname{det}\left[I_{n N}+T_{(n)}(s) \widehat{P}(s) \widehat{K}(s)\right]=\prod_{i=1}^{N} \operatorname{det}\left[I_{n}+\lambda_{i}(s) P(s) K(s)\right] .
$$

so the number of anti-clockwise encirclements of the origin made by the Nyquist plot of

$$
\operatorname{det}\left[I_{n N}+T_{(n)}(s) \widehat{P}(s) \widehat{K}(s)\right]
$$

is the same as the number of encirclements of the origin made by the Nyquist plot of

$$
\prod_{i=1}^{N} \operatorname{det}\left[I_{n}+\lambda_{i}(s) P(s) K(s)\right]
$$

which is clearly in a form simple to analyze. Now we obtain:

Corollary 1. $U(s)=\widehat{K}(s)\left(U_{\text {ref }}-Z(s)\right)$ stabilizes the system

$$
\left\{\begin{array}{l}
Y(s)=\widehat{P}(s) U(s) \\
Z(s)=H_{(n)}(s) Y(s)
\end{array}\right.
$$

iff the Nyquist plot of

$$
\prod_{i=1}^{N} \operatorname{det}\left[I_{n}+\lambda_{i}(s) P(s) K(s)\right]
$$

makes $p$ anti-clockwise encirclements of the origin, where $p$ is the number of unstable poles of

$$
\operatorname{det}[P(s) K(s)]^{N} \prod_{i=1}^{N} \lambda_{i}(s)
$$

\subsection{Robustness}

In the previous section we developed a method for analysis when homogeneous linear systems are interconnected. In the real world, the plants could be effected by nonlinearities and model errors. In this section we will propose a simple method to analyze this problem based on the framework that was presented in the previous section.

There are many perturbations models, but we will focus on two general structures that cover the low and high frequency parameter errors: 


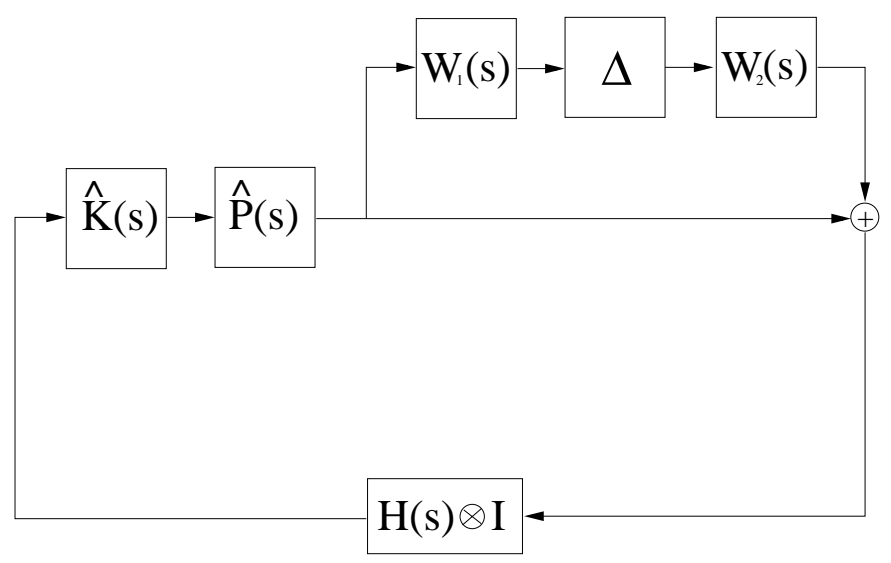

Figure 2: The interconnected system with unceratainty.

\section{The multiplicative uncertainty structure}

Let plant $i$ be perturbed by the multiplicative uncertainty $(I+$ $\left.W_{2}^{i}(s) \Delta_{i}(s) W_{1}^{i}(s)\right)$ such that the real plant dynamics is given by

$$
P_{\Delta}(s)=\left(I+W_{2}^{i}(s) \Delta_{i}(s) W_{1}^{i}(s)\right) P(s)
$$

where $W_{1}^{i}(s)$ and $W_{2}^{i}(s)$ are transfer matrices that charecterize the spatial and frequency structure of the uncertainty $\Delta_{i}(s)$ where $\left\|\Delta_{i}(s)\right\|_{\infty} \leq$ 1. Now let

$$
\begin{aligned}
\Delta(s) & =\left(\begin{array}{cccc}
\Delta_{1}(s) & 0 & \cdots & 0 \\
0 & \Delta_{2}(s) & \cdots & 0 \\
\vdots & \vdots & \ddots & \vdots \\
0 & 0 & \cdots & \Delta_{N}(s)
\end{array}\right) \\
W_{1}(s) & =\left(\begin{array}{cccc}
W_{1}^{1}(s) & 0 & \cdots & 0 \\
0 & W_{1}^{2}(s) & \cdots & 0 \\
\vdots & \vdots & \ddots & \vdots \\
0 & 0 & \cdots & W_{1}^{N}(s)
\end{array}\right)
\end{aligned}
$$

and

$$
W_{2}(s)=\left(\begin{array}{cccc}
W_{2}^{1}(s) & 0 & \cdots & 0 \\
0 & W_{2}^{2}(s) & \cdots & 0 \\
\vdots & \vdots & \ddots & \vdots \\
0 & 0 & \cdots & W_{2}^{N}(s)
\end{array}\right)
$$

Then, the total system is given by the feedback-loop shown in Figure

2. 


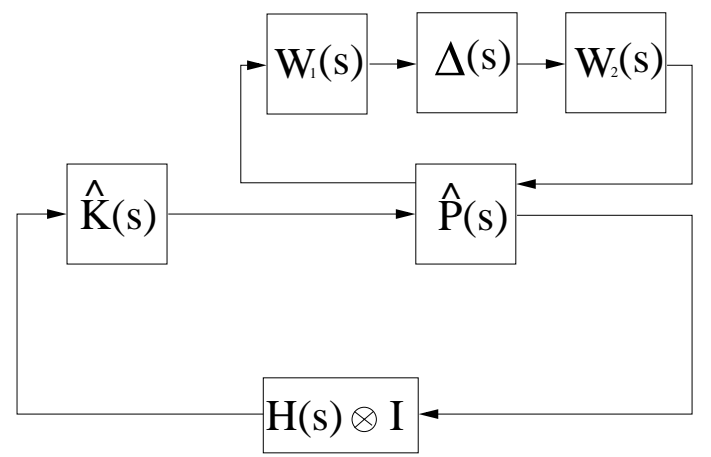

Figure 3: The interconnected system with uncertainty of the M- $\Delta$ structure.

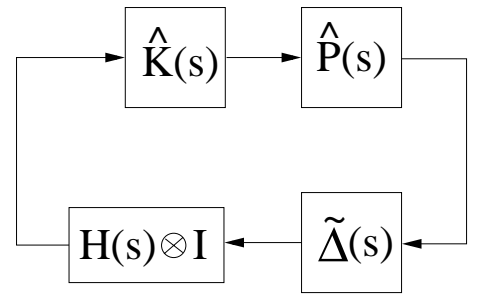

Figure 4: The interconnected system with unceratainty $\widetilde{\Delta}$.

\section{The feedback-loop structure}

Here we consider plant $i$ to be interconnected with a stable uncertainty matrix $\Delta_{i}$ where $\left\|\Delta_{i}\right\|_{\text {infty }} \leq 1$ and weighted by the stable matrices $W_{1}^{i}(s)$ and $W_{2}^{i}(s)$. Hence, the real plant dynamics are given by

$$
P_{\Delta}(s)=\left(I_{n}+P(s) W_{2}^{i}(s) \Delta_{i}(s) W_{1}^{i}(s)\right)^{-1} P(s)
$$

Constructing $\Delta(s), W_{1}(S)$, and $W_{2}(s)$ as we did in the previous perturbation model, we obtain the following equation for the transfer function of the whole system

$$
G(s)=\left(I_{n N}+H_{(n)}(s) \tilde{\Delta}(s) \widehat{P}(s) \widehat{K}(s)\right)^{-1} H_{(n)}(s) \tilde{\Delta}(s) \widehat{P}(s) \widehat{K}(s)
$$

which is similar to the previous model except that

$$
\tilde{\Delta}(s)=\left(I_{n N}+\widehat{P}(s) W_{2}(s) \Delta(s) W_{1}(s)\right)^{-1} .
$$

The feedback loop is shown in Figure 3. Both perturbation models introduced a new uncertainty $\widetilde{\Delta}$ that could be introduced as a separate block, i.e. $\widehat{P}_{\Delta}(s)=\widetilde{\Delta} \widehat{P}$ and the new loop becomes like in Figure 4. 


\section{Time-delays}

An important issue of robust stability is the problem with timedelays. The interconnected systems could have some kind of limitations when considering the links between different systems, including delays. We could introduce the time-delay matrix $\widetilde{\Delta}(s)=\left[c_{i j} e^{-s \tau_{i j}}\right]$ to represent the time-delays between different links. Another way is to include the delays in the interconnection matrix $H(s)$. The new framework for the interconnected system with perturbations included makes us tempted to use a straightforward way for stability analysis, namely the Small Gain Theorem:

Theorem 3. Proposition Consider the interconnected system in Figure 4. Assume that $\widehat{P}(s), H(s)$, and $\widetilde{\Delta}(s)$ are stable. Let

$$
\|\widetilde{\Delta}(s)\|_{\infty} \leq \beta .
$$

Then the system is stable iff

$$
\left\|H_{(n)}(s) \widehat{P}(s) \widehat{K}(s)\right\|_{\infty}<\frac{1}{\beta}
$$

We note that equation 7 is equivalent to

$$
\left\|\lambda_{i}(s) P(s) K(s)\right\|_{\infty}<\frac{1}{\beta} \quad \forall i
$$

since

$$
\begin{aligned}
\left\|H_{(n)}(s) \widehat{P}(s) \widehat{K}(s)\right\|_{\infty} & =\left\|H_{(n)}(s) \widehat{P}(s) \widehat{K}(s)\right\|_{\infty} \\
& =\left\|S_{(n)} T_{(n)}(s) \widehat{P}(s) \widehat{K}(s) S_{(n)}^{*}\right\|_{\infty} \\
& =\left\|T_{(n)}(s) \widehat{P}(s) \widehat{K}(s)\right\|_{\infty} \\
& =\left\|\lambda_{i}(s) P(s) K(s)\right\|_{\infty}
\end{aligned}
$$

Also, since $\widetilde{\Delta}(s)$ is blockdiagonal, we have that $\beta=\max \left\{\left.\left\|\widetilde{\Delta}_{i}\right\|\right|_{\infty}\right\}$.

Now we give another stability theorem for the interconnected system where we use our first result:

Theorem 4. The interconnected system in Figure 4 is stable iff

$$
\prod_{i=1}^{N} \operatorname{det}\left[I_{n}+\lambda_{i}(s) P(s) K(s)\right]
$$


makes $p$ anti-clockwise encirclements of the origin, where $p$ is the number of unstable poles of

$$
\operatorname{det}[P(s) K(s)]^{N} \prod_{i=1}^{N} \lambda_{i}(s)
$$

and $\lambda_{i}(s)$ is the ith eigenvalue of $H(s) \widetilde{\Delta}(s)$.

Of course, we don't know the eigenvalues of $H(s) \widetilde{\Delta}(s)$ since $\widetilde{\Delta}(s)$ is an uncertainty. On the other hand, it could be considered as a perturbation of the interconnection, e.i., a perturbation of the eigenvalues of $H(s)$. Tools from robust control theory might be useful to give upperbounds for these perturbations. 


\section{Applications}

Theorem 2 is stated in such a way to give as general framework as possible for the interconnection of systems with homogeneous dynamics. There are many interesting special cases that are far from being trivial when trying to use traditional techniques. But using the results of Theorem 2 enable us to analyse complicated interconnections easly.

\subsection{The Consensus Problem}

It is of great interest to make a group av plants, e.g. aerial vehicles, to reach agreement, or consensus which as it is called in Olfati-Saber and Murray[2]. We would like, for instance, to make these plants to agree on some common state or output. We want to explore how the information topology and dynamics affects the stability of the interconnected system. We will consider the problem based on the framework presented in Fax and Murray [1], but with a frequency-domain approach.

Consider a system of $N$ plants $\mathcal{P}=\left\{P_{i}\right\}^{N}$ such that each plant has $m$ inputs and $n$ outputs. Note that the assuming that the plants have the same dimensions does not imply any loss of generality. We assume that the dynamics for each plant are decoupled from the other $N-1$ plants in the system. Then we can write the system for plant $i$ in the frequency domain as

$$
Y_{i}(s)=P_{i}(s) U_{i}(s)
$$

for all $i \in\{1, \ldots, N\}$. The output $Y_{i}(s)$ is considered as a sensed information which represents the internal state measurement for plant $i$. The external state measurements $Z_{i j}(s)$ for $V_{i}$ relative to other plants is given by

$$
Z_{i j}(s)=Y_{i}(s)-Y_{j}(s), \forall j \in \mathcal{J}_{i}
$$

where $\mathcal{J}_{i} \subset\{1, \ldots, N\} \backslash\{i\}$ represents the set of plants that $P_{i}$ can sense. For simplicity, we assume that $\left|\mathcal{J}_{i}\right| \geq 1, \forall i \in\{1, \ldots, N\}$. This condition implies that each plant can sense at least one other plant. Notice that a single plant cannot drive all the terms $Z_{i j}(s)$ to zero simultaneously. Therefore, all errors must be synthesized into one signal. We introduce the new error measurement $Z_{i}(s)$ by building a weighted sum over the relative state measurements. For simplicity, we assume that the terms $Z_{i j}(s)$ are equally weighted, hence

$$
Z_{i}(s)=\frac{1}{\left|\mathcal{J}_{i}\right|} \sum_{j \in \mathcal{J}_{i}} Z_{i j}(s) .
$$


Note that this assumption does not give us a weaker result. Let $K_{i}(s)$ denote the decentralized control law for plant $i$.

Introduce

$$
U(s)=\left(\begin{array}{c}
U_{1}(s) \\
U_{2}(s) \\
\vdots \\
U_{N}(s)
\end{array}\right), \quad Y(s)=\left(\begin{array}{c}
Y_{1}(s) \\
Y_{2}(s) \\
\vdots \\
Y_{N}(s)
\end{array}\right) \quad \text { and } \quad Z(s)=\left(\begin{array}{c}
Z_{1}(s) \\
Z_{2}(s) \\
\vdots \\
Z_{N}(s)
\end{array}\right) \text {. }
$$

So letting $L_{(n)}^{i}$ denote the $i$ th row of $L_{(n)}$, we see that

$$
Z_{i}(s)=L_{(n)}^{i} Y(s)
$$

Hence, the equation for the total system is given by

$$
\left\{\begin{array}{l}
Y(s)=\widehat{P}(s) U(s) \\
Z(s)=L_{(n)} Y(s)
\end{array}\right.
$$

where $\widehat{P}(s)$ is the direct sum for the set of plants $\mathcal{P}=\left\{P_{1}(s), \ldots, P_{N}(s)\right\}$. We will explore the stability of the interconnection with plants of equal dynamics, i.e. $P_{i}(s)=P(s)$ for all $i \in\{1, \ldots, N\}$.

\subsection{Stable SISO Plants and Interconnection}

Let us consider the case when $\mathrm{P}(\mathrm{s})$ is $\mathrm{SISO}-$ stable, and the interconnection matrix $H(s)$ is stable, that is $\operatorname{det}(H(s)$ has no poles in the RHP. Then the criterion for stability of the interconnected system (1) is that the Nyquist plot of

$$
\prod_{i=1}^{n}\left(1+\lambda_{i}(s) P(s) K(s)\right)
$$

makes zero encirclements around the origin, or equivalently that the Nyquist plot of

$$
\lambda_{i}(s) P(s) K(s)
$$

makes no encirclements around $-1+0 j$, for $i=1, \ldots, n$.

Now let the interconnection matrix be the Laplacian matrix, that is $H(s)=L$. Then we see that the system is stable iff the Nyquist plot of $\lambda_{i} P(s) K(s)$ makes no encirclements around $-1+0 j$, which is equivalent to that the Nyquist plot of $P(s) K(s)$ does not encircle $-\frac{1}{\lambda_{i}}$ for $i=1, \ldots, n$. 


\subsection{Interconnection with Fixed Time-delays}

A common problem with interconnected systems is the presence of time-delays. In this section, we will find necessary and sufficient conditions, using the techniques discussed earlier.

Consider the interesting case where $H(s)=L$, that is the interconnection is given by the Laplacian matrix. Suppose that there is a fixed time-delay $\tau_{i j}$ for plant $i$ to get the sensed measurement from plant $j$ that it is connected with. Then we can write the interconnection matrix as

$$
[H(s)]_{i j}=L_{i j} e^{-\tau_{i j} s} .
$$

For instance, if the plants are SISO and stable, necessary and sufficient conditions for stability of the interconnected system is that the Nyquist plot of

$$
\prod_{i=1}^{N}\left(1+\lambda_{i}(s) P(s) K(s)\right)
$$

makes zero encirclements around the origin, where $\lambda_{i}$ is the $i$ th eigenvalue of $H(s)$. This is equivalent to that the Nyquist plot of

$$
\left.\lambda_{i}(s) P(s) K(s)\right)
$$

makes zero encirclements around $-1+0 \mathrm{j}$, for all $i$. So we can see that stability of the interconnected system depends on the structure of the interconnection given by the matrix $H(s)$, which is spanned by the topology of the interconnection(the Laplacian), and the structure of the time-delays.

A similar argument is easy to obtain for MIMO plants $P(s)$ which are not necessarily stable, using the results in sectuion 3 .

\subsection{Interconnection with Random Delays}

In this section we will assume that the plants are stable, and plant $i$ recieves the sensed measurements from plant $j$ after $\tau_{i j}$ time units. The delays $\tau_{i j}$ could be time-varying. Using the same arguments as in the fixed-delays section, we see that the interconnections matrix $H(s)$ this time has eigenvalues that are uncertain, because of the time-variation of the delays. One way is to use robustness analysis, where we consider the eigenvalues $\left\{\lambda_{i}(s)\right\}$ of $H(s)$ as perturbed, and the perturbation is given by regions that are depndent on the characteristics of variation of $\tau_{i} j$. Another way to do the analysis, which is much more conservative, is to use the Small Gain Theorem. Since we know that the entries of 


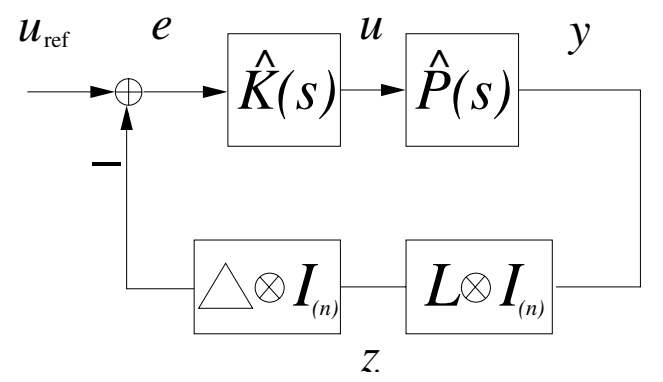

Figure 5: The interconnected system with random time-delays.

$H(s)=\left[L_{i j} e^{-\tau_{i j} s}\right]$ are characterised by the delays and Laplacian for the interconnection graph, we could easly calculate

$$
\|H(s)\|_{\infty} \leq 2 .
$$

The inequality above is obtained using the Geršgorin disc theorem. Thus, a sufficient condition is to have

$$
\|\widehat{P}(s) \widehat{K}(s)\|_{\infty}=\|P(s) K(s)\|_{\infty}<\frac{1}{2} .
$$

We illustrate the method above. Assume that plant $i$ gets information from plant $j$ telling the size of the delay. So when making the external state measurements, we can set

$$
\tau_{i}=\max \left\{\tau_{i j}, j \in \mathcal{J}_{i}\right\},
$$

and

$$
Z_{i j}=\left[Y_{i}(s)-Y_{j}(s)\right] e^{-\tau_{i} s}
$$

Thus

$$
Z(s)=\Delta L Y(s)
$$

where

$$
\Delta=\left(\begin{array}{cccc}
e^{-\tau_{1} s} & 0 & \cdots & 0 \\
0 & e^{-\tau_{2} s} & \cdots & 0 \\
\vdots & \vdots & \ddots & \vdots \\
0 & 0 & \cdots & e^{-\tau_{n} s}
\end{array}\right)
$$

Thus, using the control law $U(s)=\widehat{K}(s)\left(U_{\text {ref }}-\Delta Z(s)\right)$, we see that the system is equivalent to the one shown in Figure 5.

Let $\boldsymbol{\Delta}$ be the set of all possible time-delay connections $\Delta$, such that the closed-loop system in Figure 5 is stable. The transfer function from $U_{\text {ref }}(s)$ to $Z(s)$ is given by $(I+\Delta L \widehat{P} \widehat{K})^{-1} \Delta L \widehat{P} \widehat{K}$. This is equivalent to $L \widehat{P} \widehat{K}$ being the transfer function from $U(s)=U_{\text {ref }}(s)-\Delta Z(s)$ to $Z(s)$, that is $L \widehat{P} \widehat{K}$ is the open-loop system with the feedback system 


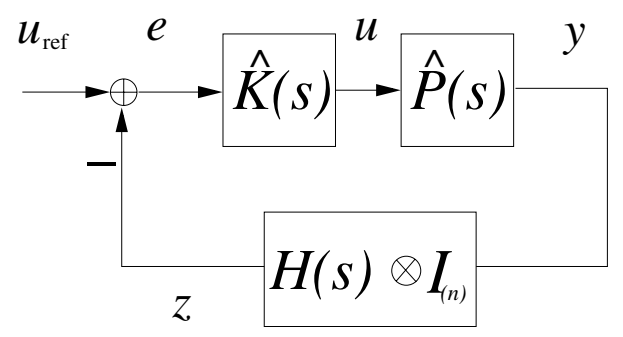

Figure 6: The interconnected system with graph uncertainty.

given by $\Delta$.

Clearly, $\|\Delta\|_{\infty}=1$. Applying the Small Gain Theorem yields that the interconnection with random time-delays is stable iff

$$
\left\|L_{(n)} \widehat{P}(s) \widehat{K}(s)\right\|_{\infty}=\left\|\lambda_{i} P(s) K(s)\right\|_{\infty}<1
$$

for all $i$, e.i.

$$
\|P(s) K(s)\|_{\infty}<\frac{1}{\left|\lambda_{i}\right|}
$$

for all $i$, where $\left\{\lambda_{i}\right\}$ are the eigenvalues of $L$.

\subsection{Time-varying Interconnection}

Itt's very interesting to explore the robustness of an interconnected system, where links between different plants could be broken or intentionally changed to achieve performance. Consider a system interconnected by the Laplacian matrix $L$. Let $\mathcal{L}$ be a set of Laplacian matrices such that the closed loop system with respect to every Laplacian $\tilde{L} \in \mathcal{L}$ is stable.

\section{Stable Plants and Multiplicative Uncertainty}

In this section we consider time-varying interconnection in the case where the plants in the system are stable. Let $\Delta$ denote the uncerttain Laplacian matrix for the system. A diagram for the closed-loop system is given by Figure 7 .

It is known that the eigenvalues for the Laplacian lie inside the unit

disc centered at $1+0 j$ (an is proof could be derived using the Geršgorin disc theorem presented in the second section). Thus $\|L\|_{\infty}=\bar{\sigma} \leq 2$. 


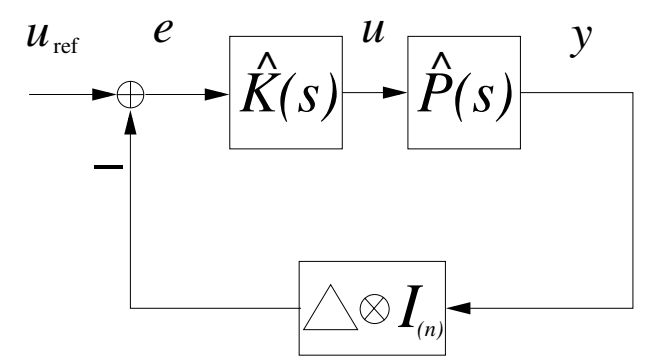

Figure 7: The interconnected system with interconnection uncertainty.

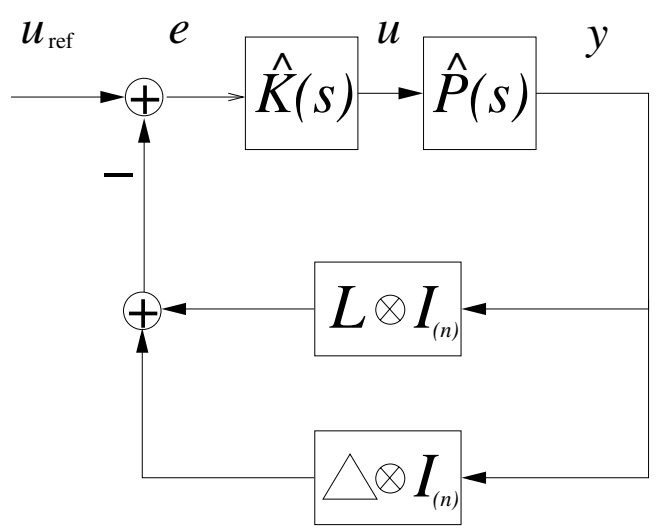

Figure 8: The interconnected system with interconnection uncertainty.

Then by the Small Gain Theorem, the interconnected system is stable iff

$$
\gamma=\|\widehat{P}(s) \widehat{K}(s)\|_{\infty}=\|P(s) K(s)\|_{\infty}<\frac{1}{2} .
$$

\section{Unstable Plants and Additive Uncertainty}

Set

$$
\Delta=\{\tilde{L}-L \mid \tilde{L} \in \mathcal{L}\}
$$

It is not hard to find that $\|\tilde{L}-L\|_{\infty} \leq 2$ using Geršgorin disc theorem. Now consider the closed-loop system shown in Figure 8: By the Small

Gain Theorem, the system is stable if

$$
\begin{aligned}
\gamma & =\left\|L \widehat{P}(s) \widehat{K}(s)(I+L \widehat{P}(s) \widehat{K}(s))^{-1}\right\|_{\infty} \\
& =\max _{i}\left\|\lambda_{i} P(s) K(s)\left(I+\lambda_{i} P(s) K(s)\right)^{-1}\right\|_{\infty}<\frac{1}{2} .
\end{aligned}
$$

There are many ways of stabilizing the system. One straightforward way is to change the feedback gain, which is simply multiplying 


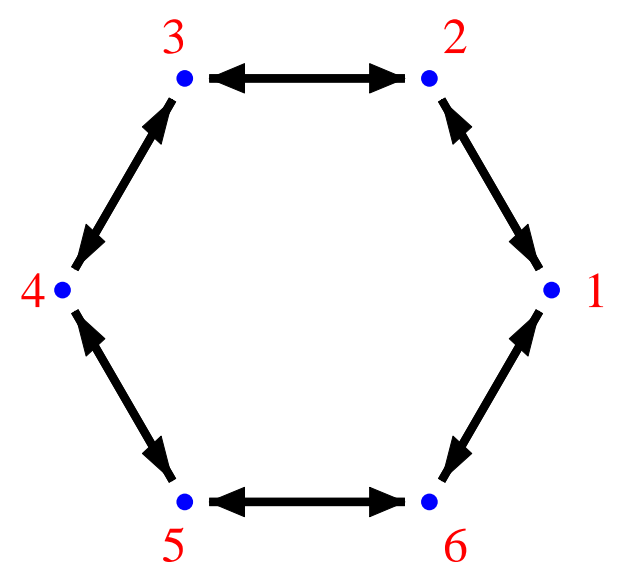

Figure 9: The graph representing the interconnection between the plants.

the Laplacian matrix $L$ with some proper real constant. Another way is to use $\mathcal{H}_{\infty}$ control to minimize $\gamma$.

\subsection{A Numerical Example}

Consider a system of 6 plants $\left\{P_{i}\right\}_{i=1}^{6}$ with equal dynamics $P(s)=$ $\frac{1}{s^{2}+a_{1} s+a_{0}}$ associated with a identical controllers $K(s)=b_{2} s^{2}+b_{1} s+b_{0}$. Suppose that plant $P_{i}$ can sense plant $P_{i+1}$ and $P_{i-1}$ for $i=1, \ldots, 6$, $P_{7}=P_{1}$ and $P_{-1}=P_{6}$. The graph representing the interconnection is given in Figure 9.

Let $\tau_{i}$ be the time-delay for $P_{i}$ to recieve the sensed signal of $P_{i+1}$ and $P_{i-1}$. Then building the relative measurement

$$
Z_{i j}=\left[Y_{i}(s)-Y_{i+1}(s)\right] e^{-\tau_{i} s}
$$

gives us the following interconnection matrix for the system(compare with the problem setup):

$$
H(s)=\left(\begin{array}{ccccc}
e^{-\tau_{1} s} & -\frac{1}{2} e^{\tau_{1} s} & 0 & \cdots & -\frac{1}{2} e^{\tau_{1} s} \\
-\frac{1}{2} e^{\tau_{2} s} & e^{-\tau_{2} s} & -\frac{1}{2} e^{\tau_{2} s} & \cdots & 0 \\
\vdots & \vdots & \vdots & \ddots & \vdots \\
-\frac{1}{2} e^{\tau_{6} s} & 0 & -\frac{1}{2} e^{\tau_{6} s} & \cdots & e^{-\tau_{6} s}
\end{array}\right)
$$

Checking the Nyquist-like plot we see that the net encirclement of the 


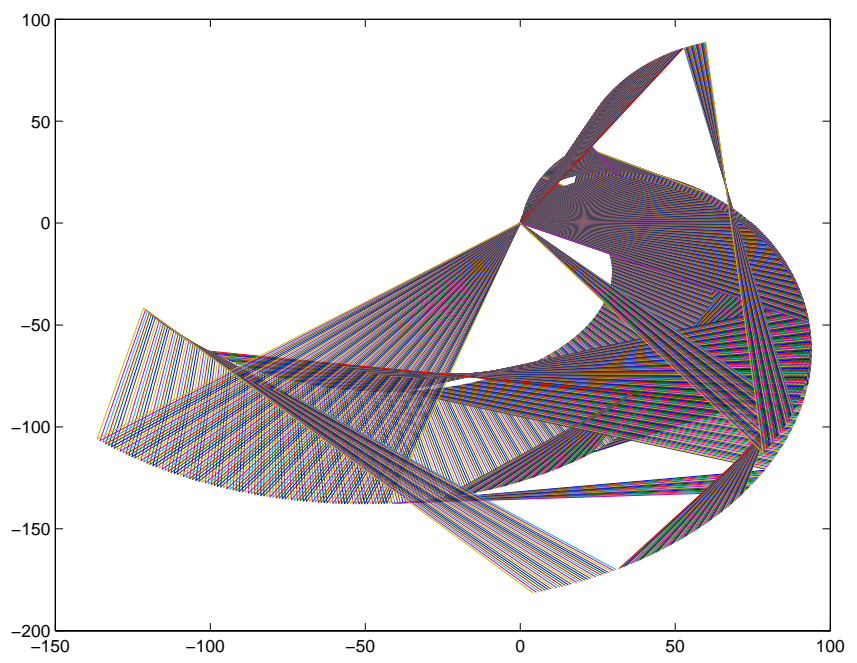

Figure 10: The Nyquist-like plot of the interconnected system .

origin is zero, hence the system must be stable.

The simulation result of the system is given in Figure 12 .

Now consider the case when we switch from the Laplacian above to another Laplacian given by Figure 13. Choosing a controller such that

$$
\|L \widehat{K}(s) \widehat{P}(s)\|_{\infty}=\left\|\lambda_{i} K(s) P(s)\right\|_{\text {infty }}<1
$$

guarentees stability, as seen in the simulation result in Figure 14. 


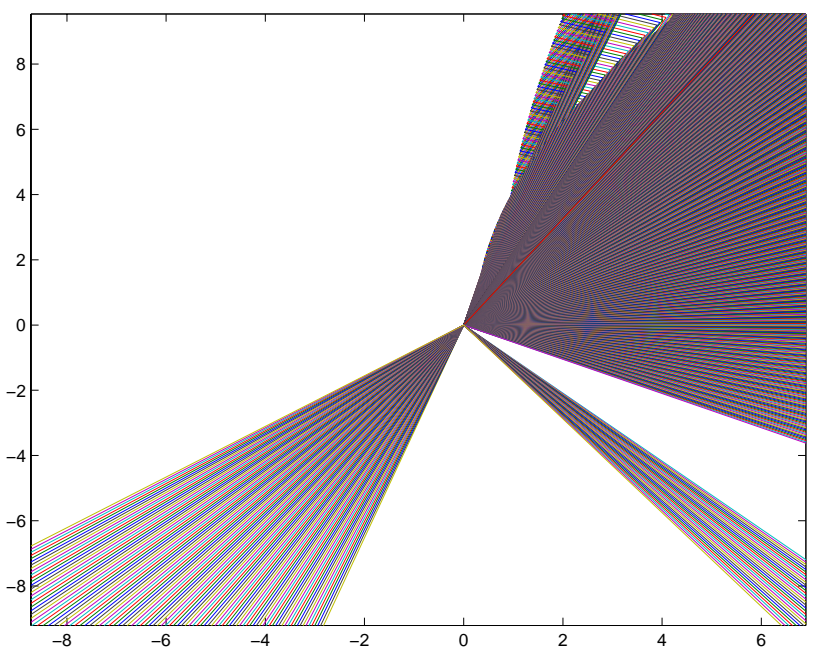

Figure 11: The Nyquist-like plot of the interconnected system zoomed around $-1+0 \mathrm{j}$.

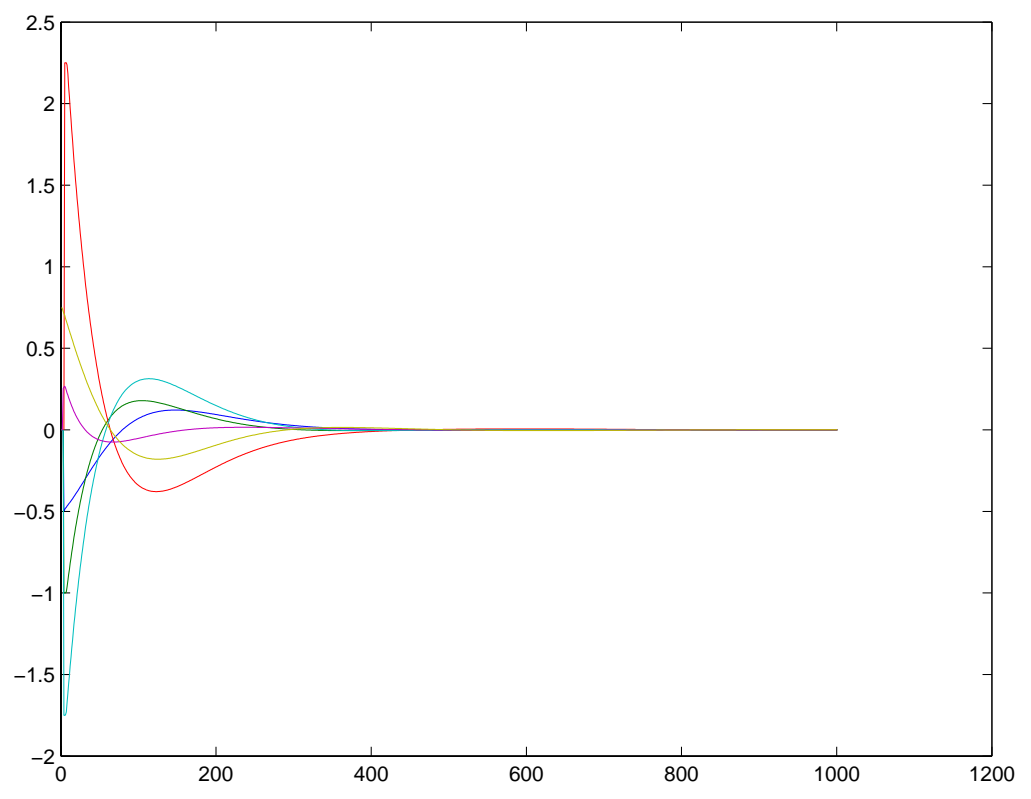

Figure 12: Simulation results of the interconnected system. 


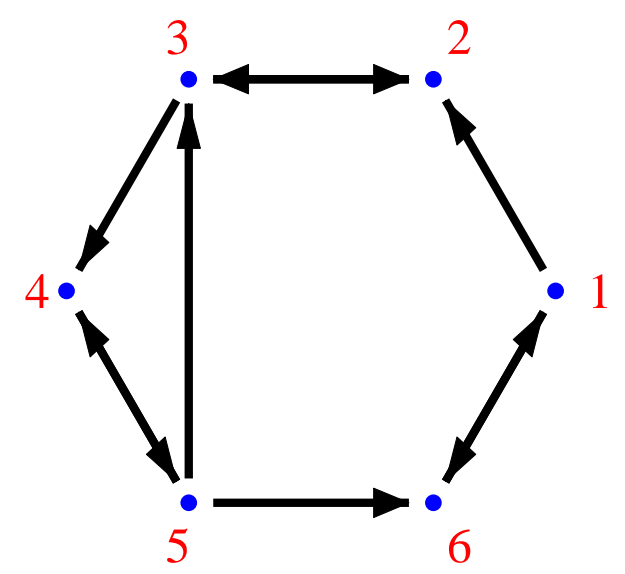

Figure 13:

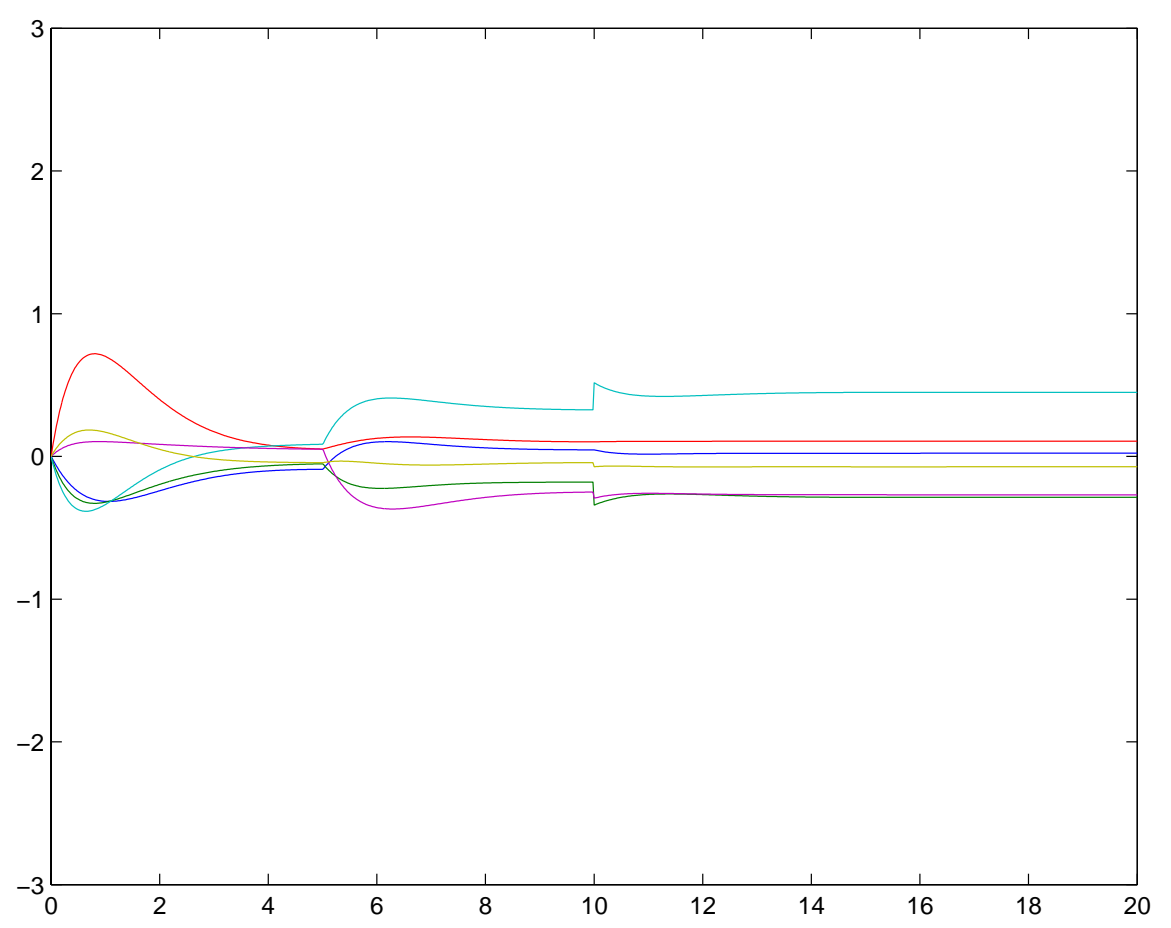

Figure 14: Switching between two topologies of the interconnection 


\section{Conlclusions and Future Work}

In this paper a framework for interconnected systems was extended from the one introduced in Fax and Murray [1].

An efficient Nyquist-like method for stability check is developed for homogeneous and almost homogeneous interconnected systems with arbitrary connection. We have seen that many problems that arises in the context of system networks could be modelled in way that fits our general framework, e.g. time-varying interconnection and the problem with time-delays.

There is still a lot to explore. The problem where the plants are heterogeneous is still important to analyze. It is very interesting to find out whether there is a similar separation principle when the plant dynamics are different. Another important issue is the problem with bounded time-varying delays. It is also of great interest to explore the role of robust control theory to obtain less conservative results and improve on the framework. There are of course more avenues of research and theory extensions where the imagination is probabely the sole limit! 


\section{A Matlab-files}

\section{A.1 Simulink Models}

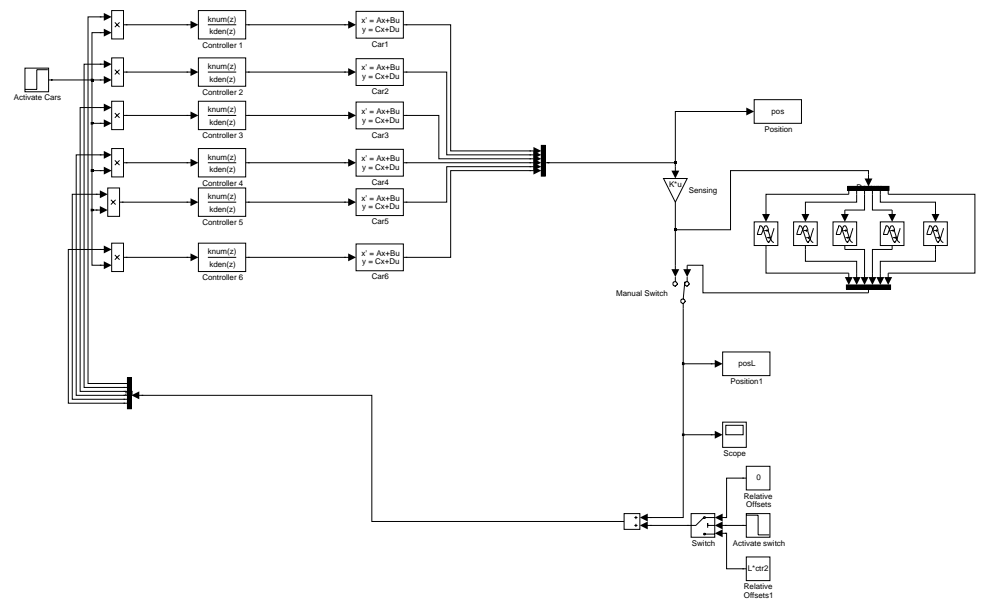

Figure 15:

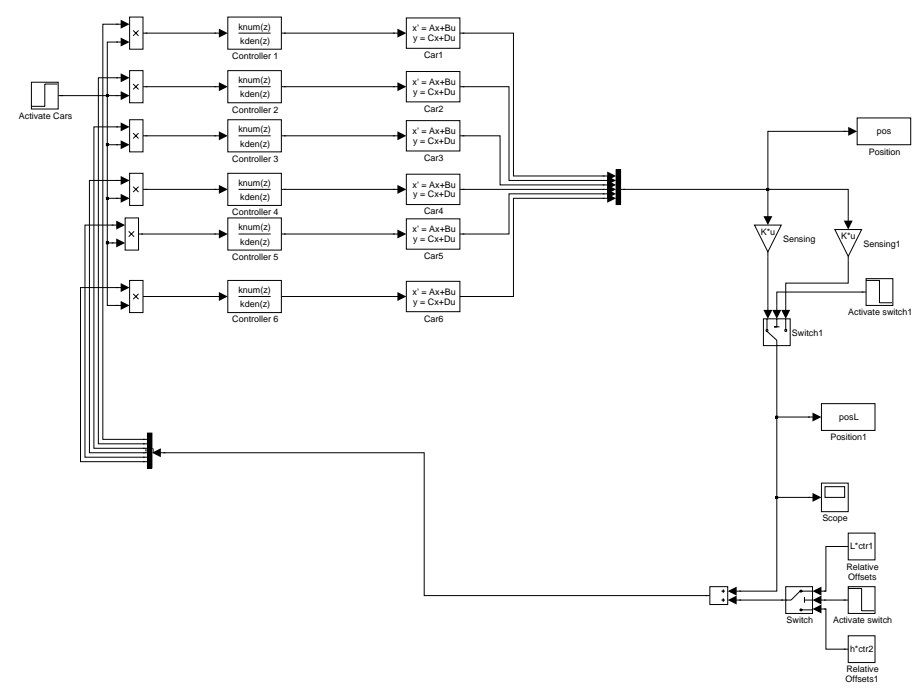

Figure 16:

\section{A.2 Matlab Code}

The matlab function below draws the Nyquist-like plots given in the Applications section. 


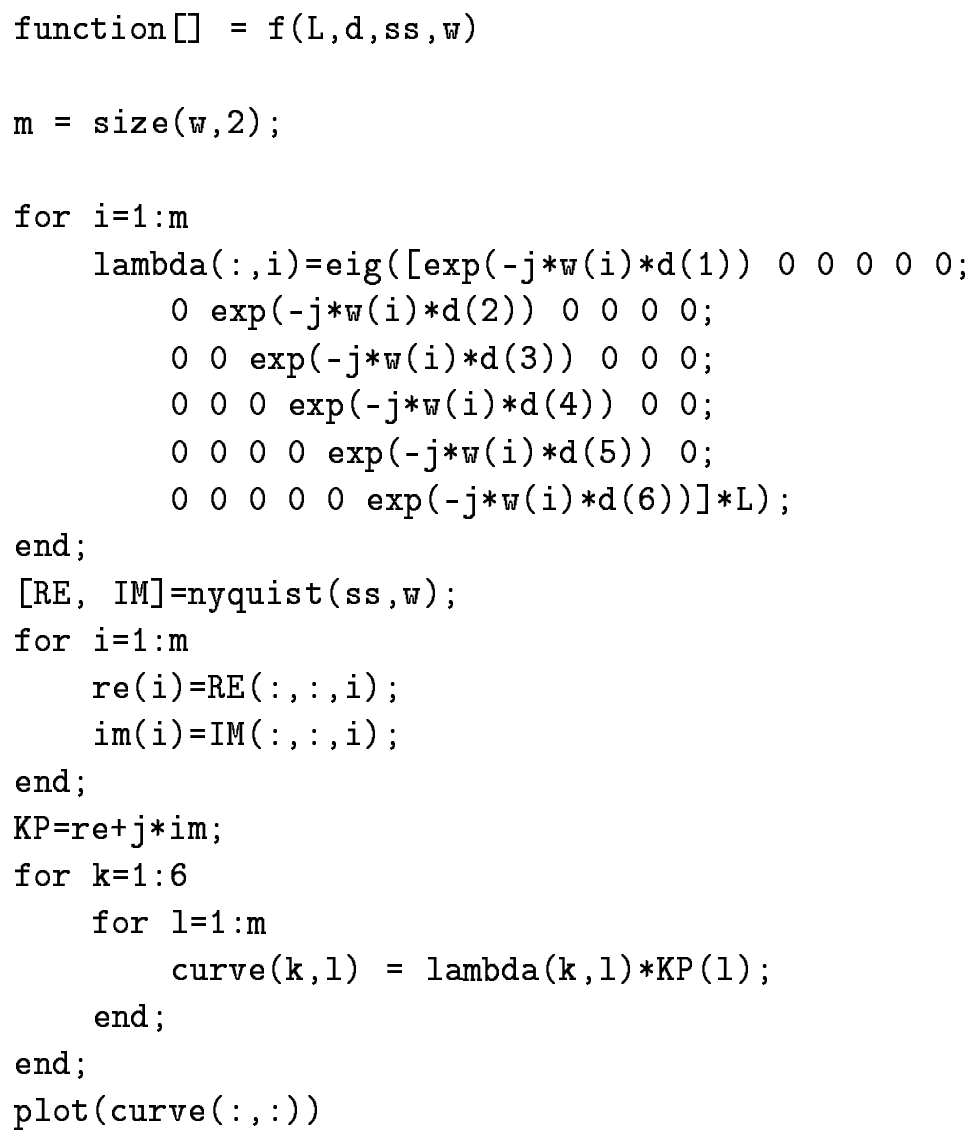




\section{References}

[1] J. A. Fax, R. M. Murray. Optimal and Cooperative Control of Vehicle Formations. PhD Thesis, California Institute of Technology, 2002.

[2] R. Olfati Saber and R. M. Murray. Consensus Protocols for Networks of Dynamic Agents. In Proc. of American Control Conference, Denver, Colorado, June 2003.

[3] V. Gupta, B. Hassibi and R. M. Murray. Stability Analysis of Stochastically Varying Formations of Dynamic Agents. In Proc. of IEEE Conference on Decision and Control, Hawaii, USA, Dec 2003.

[4] A. Jadbabaie, G. J. Pappas. Stable Flocking of Mobile Agents, Part II: Dynamic Topology. Submitted to Automatica, July 2003.

[5] L. B. Cremean and R. M. Murray. Stability analysis of interconnected nonlinear systems under matrix feedback. In Proc. of IEEE Conference on Decision and Control, Hawaii, USA, Dec 2003.

[6] G. Vinnicombe. On the stability of end-to-end congestion control for the Internet. CUED/F-INFENG/TR.398, December 2000.

[7] M. Rotkowitz and S. Lall. Descentralized Control Structures Preserved under Feedback. IEEE Conference on Decision and Control, pp. 569-575, December 2002.

[8] F. M. Callier, C. A. Desoer. Linear System Theory. Springer Ver$\operatorname{lag}(1991)$.

[9] S. Skogestad, I. Postlethwaite. Multivariable Feedback Control. John Wiley \& Sons (1997).

[10] K. Zhou, J. C. Doyle. Essentials of Robust Control. Prentice Hall (1998).

[11] C. L. Philips, R. D. Harbor. Feedback Control Systems. Prentice Hall (2000).

[12] J. Doyle, B. Franics, A. Tannenbaum. Feedback Control Theory. McMillan Publishing Co. (1990).

[13] R. Horn, C. R. Johnson. Matrix Analysis. Cambridge (1990). 